\title{
Determination of Mode Switching in Cyclic Partial Phase Transformation in Fe-0.1C-xMn Alloys as a Function of the Mn Concentration
}

\author{
HUSSEIN FARAHANI ${ }^{1,2,5}$ WEI XU, ${ }^{3,6}$ \\ and SYBRAND VAN DER ZWAAG ${ }^{1,4,7}$
}

1.-Novel Aerospace Materials Group, Faculty of Aerospace Engineering, Delft University of Technology, Delft, The Netherlands. 2.-Department of Materials Science and Engineering, Delft University of Technology, Delft, The Netherlands. 3.-State Key Laboratory of Rolling and Automation, Northeastern University, Shenyang 110819, China. 4.-School of Materials Science and Engineering, Tsinghua University, Beijing, China. 5.-e-mail: H.Farahani@ tudelft.nl. 6.—e-mail: xuwei@ral.neu.edu.cn. 7.—e-mail: S.vanderZwaag@tudelft.nl

\begin{abstract}
Controlling the kinetics of austenite decomposition by controlled partitioning of alloying elements, in particular carbon and manganese, is the key factor for optimizing the microstructures of advanced high-strength steels. In this study, a systematic set of computational and experimental cyclic partial phase transformations in low to medium manganese steels revealed a critical manganese concentration range of 1.5-2.5 mass\% at which designated manganese partitioning at moving austenite-ferrite interfaces can be used to locally increase the effective $\mathrm{Mn}$ concentration and temporarily suspend further transformation during subsequent cooling. Most interestingly, this critical concentration only becomes visible in cases of reversed partial transformations in the intercritical regime and is un-noticeable in continuous cooling or conventional isothermal treatments.
\end{abstract}

\section{INTRODUCTION}

The attractive combination of a high tensile strength with good formability in advanced highstrength steels (AHSS) is the result of tailored microstructures achieved by controlling the kinetics of the austenite decomposition. ${ }^{1-5}$ The third AHSS generation, the so-called medium manganese steels, are compositionally relatively simple carbon-manganese (C-Mn) steels having a multi-phase microstructure in combination with a high volume fraction of retained austenite. ${ }^{6-8}$ The amount and stability of the austenite phase can be regulated by an overall compositional optimization and by adjusting the temperature and time of intercritical annealing treatment which directly affects the CMn partitioning. ${ }^{9-17}$ Understanding the partitioning behavior of alloying elements, specially $\mathrm{Mn}$, for conventional and alternative thermal cycles is a key requirement for the control of the austenite ferrite phase transformation kinetics in medium $\mathrm{Mn}$ steels. ${ }^{18-21}$
Recently, the cyclic partial phase transformation (CPPT) approach has been introduced as an novel intercritical annealing route revealing the partitioning behavior of interstitial and substitutional alloying elements at transformation interfaces during austenite to ferrite and ferrite to austenite phase transformations. $^{22,23}$ In the typical CPPT thermal route, the temperature is cycled between two temperatures in the intercritical region, such that both austenite and ferrite phases are present at all times and additional nucleation of either ferrite or austenite is proved to be absent. The observed complex transformation kinetics during CPPT experiments in low $(<0.5$ mass $\%) \mathrm{Mn}$ steels and medium Mn steels can be completely explained by considering local partitioning of $\mathrm{Mn}$ at the mobile interfaces. ${ }^{24}$ As the kinetics of cyclic phase transformations in steels with higher Mn levels (up to 5 mass \%) has been observed to be rather different from that of steels with lower Mn levels, ${ }^{25}$ it is interesting to obtain more insight into the origin of these differences and to determine the role of the 
Mn partitioning on the transitions in the transformation kinetics. To this aim, the present work focuses on the transformation kinetics during and immediately after the cyclic transformation cycle as a function of the Mn concentration. The work presented here involves both computational simulations and complementary dilatometry experiments.

The local equilibrium (LE) model was used to simulate cyclic partial phase transformations in a series of alloys with compositions of Fe-0.1C- $x \mathrm{Mn}$ $(x=0.1-3.0$ mass\%; details shown in Table I). In the LE model, interfacial concentrations of $\mathrm{C}$ and $\mathrm{Mn}$ in ferrite and austenite phases are selected on the basis of an assumed equal chemical potential for each phase across the interface. The current simulations were made using Dictra (linked to TCFE7 and MOB2 databases ${ }^{26}$ ) and assumed a 1D-geometry with a size of $25 \mu \mathrm{m}$ presumed to represent an austenite grain size of $50 \mu \mathrm{m}$. Using this geometry, all the important transitions in local interface velocity as a function of the (complex) thermal history can be captured and the transformation curves in all aspects are comparable to those obtained in dilatometry experiments. ${ }^{23,27,28}$

Simulations were carried out for the three thermal routes shown schematically in Fig. $1 \mathrm{a}, \mathrm{b}$ and $\mathrm{c}$. The actual simulations start from point A (see Fig. 1) where a fully austenitic microstructure is present. All subsequent cooling or heating rates (CR) are $1 \mathrm{~K} / \mathrm{s}$. In the CPPT route (Fig. 1a), the temperature is cycled between $T_{1}$ and $T_{2}$ and held at each temperature for $1200 \mathrm{~s}(20 \mathrm{~min})$ and then cooled further to room temperature. In the continuous cooling (CC) route (Fig. 1b), the temperature is decreased continuously from $T_{2}$. In the isothermal holding + cooling (IC) route (Fig. 1c) after cooling from $T_{2}$ to $T_{1}$, the temperature is held constant for a time equal to the total intercritical holding time in the CPPT route, and then the temperature is continuously decreased. The $T_{1}$ and $T_{2}$ temperatures in the cyclic transformations for each composition are selected taking into account the corresponding Ae1 and Ae3 temperatures calculated with ThermoCalc, ${ }^{29}$ and imposing the requirement of a fixed cycling range $\Delta T=T_{2}-T_{1}=50 \mathrm{~K}$ and comparable equilibrium fractions of ferrite $\left(f_{\mathrm{eq}}^{\alpha}\right)$ at $T_{1}$ and $T_{2}$ for all compositions. The selected $T_{1}$ and $T_{2}$ values and the equilibrium fractions as predicted by ThermoCalc are listed in Table I against the sample code.

Figure 2a, b, c, d, e, f, $\mathrm{g}$ and $\mathrm{h}$ shows the simulation results for the CPPT (solid lines), CC (dotted lines) and IC (dashed lines) thermal routes for compositions Fe-0.1C- $x \mathrm{Mn}(x=0.1-3.0$ mass $\%)$, respectively. The results of the simulations are presented as graphs of interface position versus temperature. Given the 1D nature of the model, the position of the interface represents the ferrite fraction present (with an interface position of $25 \mu \mathrm{m}$ corresponding to $100 \%$ ferrite). For the cooling rate applied, the CC curves for all the compositions below 2 mass\% $\mathrm{Mn}$ show ample and smooth migration of the interface but only minute amounts of interface migration for higher $\mathrm{Mn}$ concentrations, indicating significantly less formation of intercritical ferrite.

The IC curves in Fig. 2a, b, c, d, e, f, g and h show variations in the interface migration behavior with increasing $\mathrm{Mn}$ concentration in a similar trend to that of the CC curves. While for systems with $\mathrm{Mn}$ content below 2 mass\% a significant ferrite formation during the isothermal holding stage is predicted, for higher Mn content the total fraction of isothermally formed ferrite reduces significantly. More importantly, for all Mn concentrations, the migration of the interface at the end of the isothermal holding step picks up as soon as the final cooling starts and the behavior resembles that of the CC curves for that particular alloy.

For the CPPT curves, a rather different behavior as a function of the Mn concentration is predicted. For Mn concentrations below 0.5 mass\% (Fig. 1a and b), a very short so-called 'stagnant stage ${ }^{23}$ is observed during which only minimal austeniteferrite interface migration is predicted to occur. The CPPT curves following the cyclic treatment show a continued migration of the interface when the final

Table I. The cyclic transformation temperatures of $T_{1}$ and $T_{2}$ and the equilibrium fraction of ferrite predicted by ThermoCalc ${ }^{29}$ for all the simulated composition systems

\begin{tabular}{|c|c|c|c|c|c|}
\hline Alloy tag & Composition (all in mass\%) & $T_{1}$ & $\underline{\mathbf{f}_{\mathrm{eq}}^{\alpha} \text { at } T_{1}}$ & $\boldsymbol{T}_{\mathbf{2}}$ & $\underline{\mathbf{f}_{\mathbf{e q}}^{\alpha} \text { at } \boldsymbol{T}_{\mathbf{2}}}$ \\
\hline $0.1 \mathrm{Mn}$ & $\mathrm{Fe}-0.1 \mathrm{C}-0.1 \mathrm{Mn}$ & $1063 \mathrm{~K}\left(790^{\circ} \mathrm{C}\right)$ & 0.75 & $1113 \mathrm{~K}\left(840^{\circ} \mathrm{C}\right)$ & 0.44 \\
\hline $0.25 \mathrm{Mn}$ & $\mathrm{Fe}-0.1 \mathrm{C}-0.25 \mathrm{Mn}$ & $1058 \mathrm{~K}\left(785^{\circ} \mathrm{C}\right)$ & 0.74 & $1108 \mathrm{~K}\left(835^{\circ} \mathrm{C}\right)$ & 0.43 \\
\hline $0.5 \mathrm{Mn}$ & $\mathrm{Fe}-0.1 \mathrm{C}-0.50 \mathrm{Mn}$ & $1048 \mathrm{~K}\left(775^{\circ} \mathrm{C}\right)$ & 0.70 & $1098 \mathrm{~K}\left(825^{\circ} \mathrm{C}\right)$ & 0.43 \\
\hline $1 \mathrm{Mn}$ & Fe-0.1C-1.0Mn & $1033 \mathrm{~K}\left(760^{\circ} \mathrm{C}\right)$ & 0.73 & $1083 \mathrm{~K}\left(810^{\circ} \mathrm{C}\right)$ & 0.4 \\
\hline $1.5 \mathrm{Mn}$ & Fe-0.1C-1.5Mn & $1018 \mathrm{~K}\left(745^{\circ} \mathrm{C}\right)$ & 0.70 & $1068 \mathrm{~K}\left(795^{\circ} \mathrm{C}\right)$ & 0.38 \\
\hline $2 \mathrm{Mn}$ & Fe-0.1C-2.0Mn & $1003 \mathrm{~K}\left(730^{\circ} \mathrm{C}\right)$ & 0.69 & $1053 \mathrm{~K}\left(780^{\circ} \mathrm{C}\right)$ & 0.36 \\
\hline $2.5 \mathrm{Mn}$ & $\mathrm{Fe}-0.1 \mathrm{C}-2.5 \mathrm{Mn}$ & $988 \mathrm{~K}\left(715^{\circ} \mathrm{C}\right)$ & 0.67 & $1038 \mathrm{~K}\left(765^{\circ} \mathrm{C}\right)$ & 0.34 \\
\hline $3 \mathrm{Mn}$ & $\mathrm{Fe}-0.1 \mathrm{C}-3.0 \mathrm{Mn}$ & $973 \mathrm{~K}\left(700^{\circ} \mathrm{C}\right)$ & 0.65 & $1023 \mathrm{~K}\left(750^{\circ} \mathrm{C}\right)$ & 0.32 \\
\hline
\end{tabular}



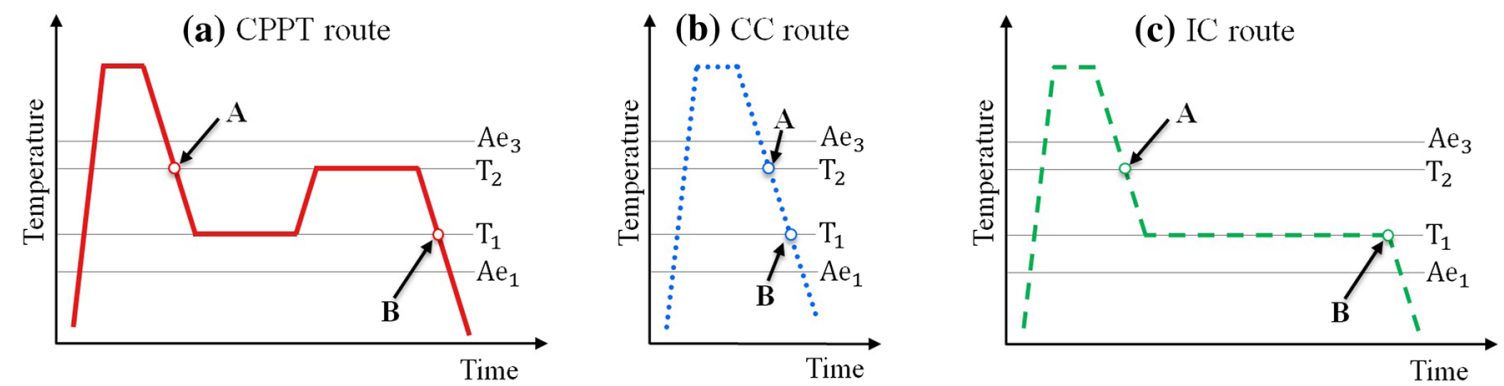

Fig. 1. Schematics of typical (a) cyclic partial phase transformation (CPPT), (b) continuous cooling (CC) and (c) isothermal holding and cooling (IC) thermal routes. $A$ marks the condition to start the simulations and $B$ the condition for which the calculated Mn profiles are presented in Fig. 3.

cooling below the $T_{1}$ temperature starts, and a behavior is observed which resembles that for the $\mathrm{CC}$ and IC treatments.

For systems with $\mathrm{Mn}$ concentrations from 0.5 mass\% to 1.5 mass\% (Fig. 2c, $\mathrm{d}$ and e), a clear stagnant stage both in the austenite to ferrite and the ferrite to austenite transformations can be distinguished in the CPPT curves. ${ }^{23}$ For the $0.5 \mathrm{Mn}$ and $1 \mathrm{Mn}$ systems, the stagnant stage in the ferrite formation when cooling form the $T_{2}$ temperature and finishes before the lower cycling temperature $T_{1}$ and the interface continues to migrate smoothly during final cooling from $T_{1}$ similar to the $\mathrm{CC}$ and IC curves. In a different way for the $1.5 \mathrm{Mn}$ system, the stagnant stage in ferrite formation continues until below $T_{1}$, generating a post-cyclic stagnant stage in the interface migration which is absent in the corresponding curves of the CC and IC routes.

For systems with $\mathrm{Mn}$ concentration above 2 mass\% (Fig. 2f, $g$ and $h$ ), the CPPT curves primarily show stagnant stages, and the isothermal direct formation of ferrite or austenite is negligible. Furthermore, for the $2 \mathrm{Mn}$ and $2.5 \mathrm{Mn}$ systems, the austenite to ferrite phase transformation during final cooling from $T_{1}$ is fully suppressed to well below the $T_{1}$ temperature. For the $3 \mathrm{Mn}$ system, migration of the interface during both CPPT, CC and IC thermal routes is minimal and the marginal differences between curves for all three thermal routes predict the formation of negligible amounts of ferrite when subjected to any of the three thermal routes.

The observed transitions in cyclic behavior of the simulated systems can be explained by taking into account the decisive effect of local Mn concentrations on the transformation kinetics. Figure $3 a, b, c$, $\mathrm{d}, \mathrm{e}, \mathrm{f}, \mathrm{g}$ and $\mathrm{h}$ shows the local Mn profile in relevant regions near the moving austenite-ferrite interface for all of the simulated systems at $T_{1}$ during cooling after the CPPT, CC and IC treatments (point B in Fig. 1a, b and c). The differences in the Mn profile can explain well the kinematic features in the interface migration behavior by considering the transition from negligible partitioning local equilibrium (NPLE) to partitioning local equilibrium (PLE) mode at transformation interfaces..$^{1,23,30,31}$ The Mn concentration profiles after the CC and IC treatments show a Mn spike at the interface position as a result the LE condition at the interface. The position of these Mn spikes is consistent with the positions of the interface in the corresponding curves in Fig. 2. For all nominal Mn concentrations, the $\mathrm{Mn}$ profile at $T_{1}$ at the end of the CPPT treatment is quite different from the profiles after $\mathrm{CC}$ and IC routes.

After the CPPT treatment, the systems with Mn concentration up to 1.5 mass\% (Fig. 3a, b, c, $d$ and e) show a profile Mn profile with a zig-zag shape attributed to the LE condition at the interface marking the ferrite to austenite phase transformation during isothermal holding at $T_{2}$, a $\mathrm{Mn}$ spike marking the interface position for the austenite to ferrite transformation during final cooling similar to that for the CC and IC curves, and finally an expanded spike as a result of the LE condition at the interface during isothermal austenite to ferrite phase transformation at $T_{1}$ after the first cooling stage (these features are best demonstrated in Fig. 3d).

In systems with a Mn content below 0.5 mass\%, the partitioning of $\mathrm{Mn}$ at the interface is too insignificant to cause any detectable disturbance in the interface motion and phase transformation kinetics in the three CPPT, CC and IC routes proceeding under the NPLE mode. ${ }^{32}$ For the $0.5 \mathrm{Mn}$ and $1 \mathrm{Mn}$ systems, the spikes of $\mathrm{Mn}$ formed at $T_{1}$ and $T_{2}$ are moderately strong and cause stagnant stages in the austenite to ferrite and ferrite to austenite transformations in the CPPT route. However, the interface can still move under the NPLE condition after passing the existing $\mathrm{Mn}$ spike.

In the $1.5 \mathrm{Mn}$ system, the pinning effect of $\mathrm{Mn}$ spike partly limits the back migration of the interface during austenite reversion at $T_{2}$, such that the existing expanded $\mathrm{Mn}$ spike formed at $T_{1}$ and the zig-zag profile formed at $T_{2}$ overlap and create an enriched Mn region (see Fig. 3e), leading to a local increase of the effective Mn concentration. Passage of the interface (during the final cooling stage from $T_{1}$ ) through this locally enriched $\mathrm{Mn}$ region requires severe partitioning of $\mathrm{Mn}$ at the interface (similar to the PLE mode), and consequently causes the post- 

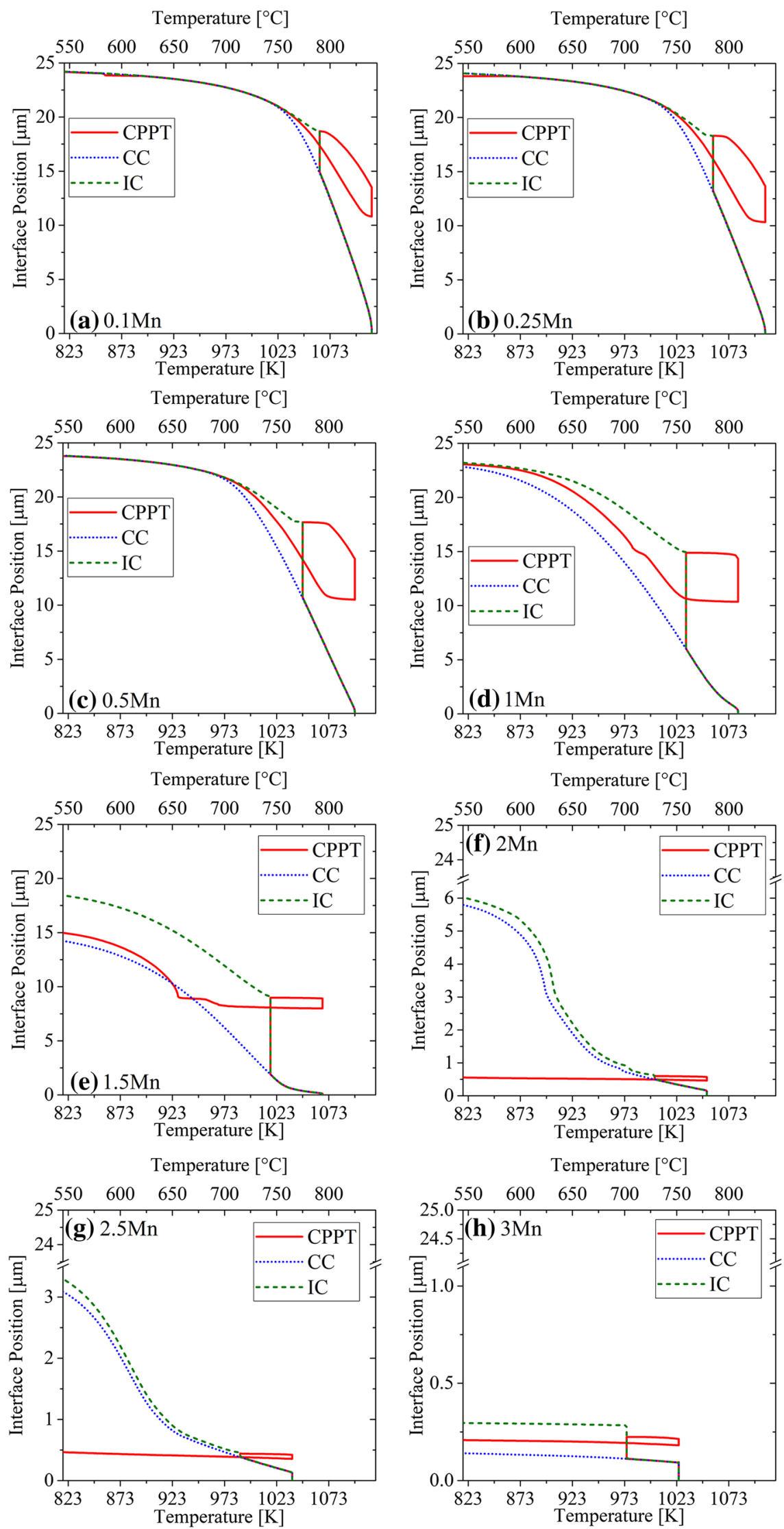

Fig. 2. Simulation results of CPPT (solid lines), CC (dotted lines) and IC (dashed lines) thermal routes using the LE model in systems of (a) $0.1 \mathrm{Mn}$, (b) $0.25 \mathrm{Mn}$, (c) $0.5 \mathrm{Mn}$, (d) $1 \mathrm{Mn}$, (e) $1.5 \mathrm{Mn}$, (f) $2 \mathrm{Mn},(\mathrm{g}) 2.5 \mathrm{Mn}$ and (h) $3 \mathrm{Mn}$, as indicated in Table I. 
Determination of Mode Switching in Cyclic Partial Phase Transformation in Fe-0.1C- $x \mathrm{Mn}$ Alloys as a Function of the Mn Concentration
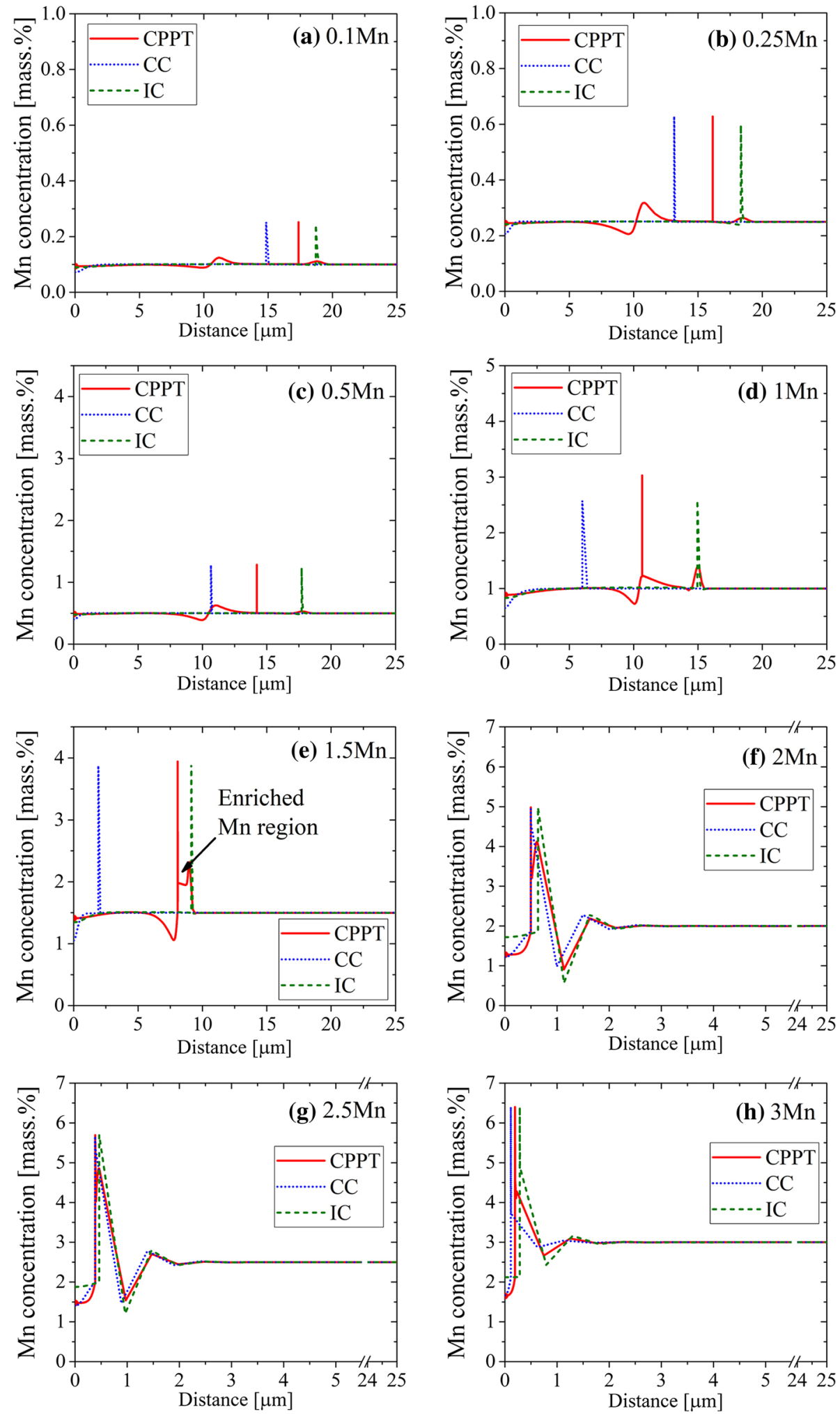

Fig. 3. Mn profile after simulation of CPPT (solid lines), CC (dotted lines) and IC (dashed lines) thermal routes (point $B$ in Fig. 1) using the LE model in systems of (a) $0.1 \mathrm{Mn}$, (b) $0.25 \mathrm{Mn}$, (c) $0.5 \mathrm{Mn}$, (d) $1 \mathrm{Mn}$, (e) $1.5 \mathrm{Mn}$, (f) $2 \mathrm{Mn}$, (g) $2.5 \mathrm{Mn}$ and (h) $3 \mathrm{Mn}$ as indicated in Table I.

cyclic stagnation in ferrite formation. As no such $\mathrm{Mn}$ spikes are created in the CC and IC treatments, the kinetic curves show no stagnation upon final cooling.
For the systems with 2 mass $\% \mathrm{Mn}$ and more (Fig. 3f, $g$ and h), the phase transformations involve full $\mathrm{Mn}$ partitioning at the (slowly moving) interfaces (PLE mode). Due to the severe pinning effect 
of the Mn spike formed at interface and the marginal migration of the interface during CPPT, the three peaks in the Mn profile (as observed in lower Mn systems after CPPT) virtually overlap and are no longer distinguishable. Thus, the Mn profile after CPPT is very similar to that of the CC and IC routes, but only in the CPPT, and final cooling starts with the interface positioned behind the very narrow but highly enriched $\mathrm{Mn}$ region that can seriously halt the migration of the interface during final cooling after the CPPT route.

The predicted stagnation in ferrite formation in lean Mn steels with Mn concentrations around 1.01.5 wt.\% Mn during stages of the CPPT treatment has been confirmed experimentally using dilatometry ${ }^{33}$ and in situ confocal microscopy. ${ }^{34}$ However, in order to assess the predicted trends in the transformation kinetics for the three thermal treatments as a function of the $\mathrm{Mn}$ concentration, a series of ternary alloys with different $\mathrm{Mn}$ concentrations close to those used in the computational experiments, listed in Table II, were collected. A Bähr DIL 805A/D/T Quenching Dilatomer was used to measure the longitudinal dilatation of the specimens (typically $10 \mathrm{~mm}$ length and $4 \mathrm{~mm}$ diameter), using the same thermal routes as used in the LE simulations. All specimens were heated with a rate of $10 \mathrm{~K} / \mathrm{s}$ to $1273 \mathrm{~K}\left(1000^{\circ} \mathrm{C}\right)$ and kept for $300 \mathrm{~s}(5 \mathrm{~min})$ to ensure full austenitization. Then, the samples were subjected to the CC, IC and CPPT thermal routes with a constant rate of $1 \mathrm{~K} / \mathrm{s}$ under vacuum condition with a maximum pressure of $5 \times 10^{-4}$ mbar. Fresh samples were used for each different thermal route. For alloys with a $\mathrm{Mn}$ concentration below 2 mass\%, the CPPT route was performed using isothermal intercritical holdings of $1200 \mathrm{~s}$ (20 min) similar to the simulations. For alloys with $\mathrm{Mn}$ levels above 2 mass\%, the cyclic treatments were carried out with isothermal holding at $T_{1}$ for $86,400 \mathrm{~s}(24 \mathrm{~h})$ and at $T_{2}$ for $7200 \mathrm{~s}$ $(2 \mathrm{~h})$ to compensate for the very slow rate of nucleation and to reach a starting ferrite fraction comparable to that in the simulations.

Figure $4 \mathrm{a}, \mathrm{b}, \mathrm{c}, \mathrm{d}, \mathrm{e}, \mathrm{f}, \mathrm{g}$ and $\mathrm{h}$ shows the measured longitudinal dilatation curves for the experiments using the CC (dotted lines), IC (dashed lines) and

Table II. Composition of the alloys used for CPPT and $\mathrm{CC}$ experiments

\begin{tabular}{lc}
\hline Alloy tag & Composition (all in mass\%) \\
$0.15 \mathrm{Mn}$ & $\mathrm{Fe}-0.023 \mathrm{C}-0.17 \mathrm{Mn}$ \\
$0.25 \mathrm{Mn}$ & $\mathrm{Fe}-0.095 \mathrm{C}-0.25 \mathrm{Mn}$ \\
$0.5 \mathrm{Mn}$ & $\mathrm{Fe}-0.0848 \mathrm{C}-0.47 \mathrm{Mn}$ \\
$1 \mathrm{Mn}$ & $\mathrm{Fe}-0.095-1.1 \mathrm{Mn}$ \\
$1.5 \mathrm{Mn}$ & $\mathrm{Fe}-0.061 \mathrm{C}-1.52 \mathrm{Mn}$ \\
$2 \mathrm{Mn}$ & $\mathrm{Fe}-0.135 \mathrm{C}-2.058 \mathrm{Mn}$ \\
$2.5 \mathrm{Mn}$ & $\mathrm{Fe}-0.06 \mathrm{C}-2.4 \mathrm{Mn}$ \\
$3 \mathrm{Mn}$ & $\mathrm{Fe}-0.1 \mathrm{C}-3.0 \mathrm{Mn}$ \\
\hline
\end{tabular}

the CPPT (solid lines) thermal routes. The CC dilatation curves for all the $\mathrm{Mn}$ concentrations indicate steady austenite decomposition, while the dilatation curves for the IC and CPPT routes show distinctly different behavior depending on the Mn concentration. It is worth mentioning that the stagnant stages in the experimental dilatation curves can be identified by the linear contraction or expansion of the specimens during cooling or heating.

The observed experimental transformation curves plotted Fig. 4 are all in very good agreement with their respective predictions made by the LE model. In line with the simulations, the experimental CPPT curves for $0.15 \mathrm{Mn}, 0.25 \mathrm{Mn}, 0.5 \mathrm{Mn}$ and $1 \mathrm{Mn}$ alloys (Fig. 4a, b, c and d) show direct transformation and stagnant stages with lengths up to $50 \mathrm{~K}$ during the thermal cycles. For these alloys, no postcyclic stagnation in the ferrite formation after the cyclic treatment is observed. The IC curves in these alloys indicate significant ferrite formation during isothermal holding. The minor non-linearity in the IC dilatation curves during the final cooling are an indication of the transformation of the small austenite fraction present after isothermal holding.

For the $1.5 \mathrm{Mn}$ alloy (Fig. 4e), the IC curve shows significant ferrite formation both during isothermal holding and final cooling. In the CPPT curve, the isothermal direct ferrite formation and austenite reversion stages during the isothermal holdings at $T_{1}$ and $T_{2}$, respectively, are easily identified. The predicted post-cyclic stagnant stage in the ferrite formation is clearly present in the experimental CPPT curve where, after the austenite reversion at $T_{2}$, the ferrite formation is suppressed for about $40 \mathrm{~K}$ of cooling below $T_{1}$ (when compared to the CC curve). This stage is obviously absent in the experimental CC and IC curves.

For the alloys with 2 mass\% of $\mathrm{Mn}$ and higher (Fig. 4f, $\mathrm{g}$ and $\mathrm{h}$ ), due to extended isothermal holding times in the CPPT and IC routes, the austenite decomposition starts at higher temperatures compared to the CC routes. As predicted by the simulations, the cyclic partial phase transformations in these alloys proceed with only slight isothermal transformations during holdings at $T_{1}$ and $T_{2}$ temperatures. In the $2 \mathrm{Mn}$ and $2.5 \mathrm{Mn}$ alloys, the CPPT treatment causes a post-cyclic stagnant stage in austenite decomposition during cooling below $T_{1}$ for about $25 \mathrm{~K}$ (when compared to the corresponding CC curves). Although ferrite, and therefore the austenite-ferrite interfaces, are present at the end of both the CPPT and the IC routes, the post-cyclic stagnant stage is only observed after the CPPT route and is absent in the samples subjected to the IC route. In the $3 \mathrm{Mn}$ alloy, showing a very good agreement with the predictions by the LE model, it is clear that the CPPT treatment has almost no effect on the transformation kinetics during final cooling which proceeds in a similar manner in the CC, IC and CPPT treated samples. 
Determination of Mode Switching in Cyclic Partial Phase Transformation in Fe-0.1C- $x \mathrm{Mn}$ Alloys as a Function of the Mn Concentration
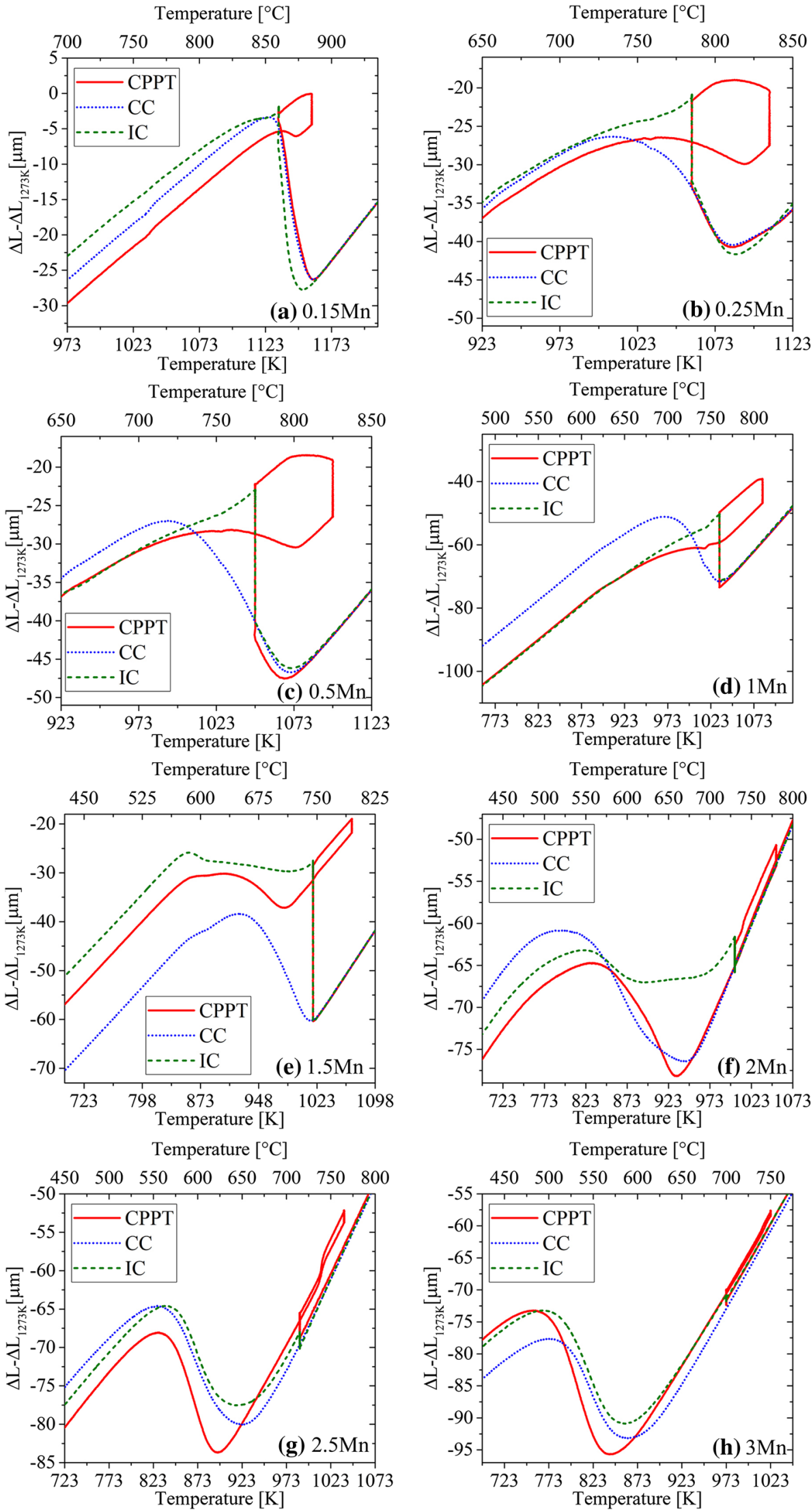

Fig. 4. Experimental dilatation results of CPPT (solid lines), CC (dotted lines) and IC (dashed lines) thermal routes in alloys with (a) $0.15 \mathrm{Mn}$, (b) $0.25 \mathrm{Mn},(\mathrm{c}) 0.5 \mathrm{Mn},(\mathrm{d}) 1 \mathrm{Mn},(\mathrm{e}) 1.5 \mathrm{Mn},(\mathrm{f}) 2 \mathrm{Mn},(\mathrm{g}) 2.5 \mathrm{Mn}$ and (h) $3 \mathrm{Mn}$ as indicated in Table II. 


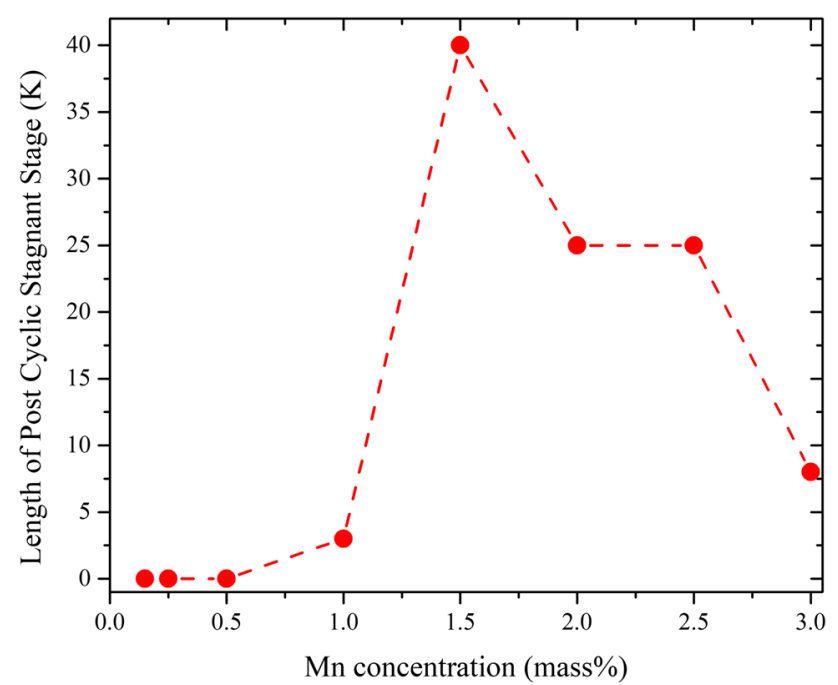

Fig. 5. Length of experimentally measured post-cyclic stagnant stage after the CPPT treatments versus the Mn concentration.

The length of experimentally measured post cyclic stagnant stage in the Mn containing alloys with different $\mathrm{Mn}$ concentrations is shown in Fig. 5. These experimental results identify a critical $\mathrm{Mn}$ concentration range from about $1.5-2.5$ mass $\%$ in which formation of the intercritical and post-intercritical ferrite can be suppressed significantly via austenite reversion through the cyclic treatment. The CPPT treatment in these range of composition can locally increase the effective Mn concentration and enhance stabilization of retained austenite with suppression of further intercritical ferrite formation.

Comparing the experimental and computationally simulated CPPT curves for the $1.5 \mathrm{Mn}, 2 \mathrm{Mn}$ and $2.5 \mathrm{Mn}$ systems, a difference in the length (i.e. duration) of the post-cyclic stagnant stage is observed. While the simulations predict stronger suppression of ferrite formation after CPPT for higher Mn contents, in the physical experiments, notwithstanding the extended time for ferrite nucleation in the $2 \mathrm{Mn}$ and $2.5 \mathrm{Mn}$ alloys, the post-cyclic stagnant stages in these alloys are shorter than that observed in the $1.5 \mathrm{Mn}$ alloy (Fig. 5). The difference arises from the lower fraction of intercritical ferrite (and consequently smaller number of available interfaces) formed during the first isothermal holding at $T_{1}$ in the in $2 \mathrm{Mn}$ and $2.5 \mathrm{Mn}$ alloys compared to the $1.5 \mathrm{Mn}$ alloy.

It is worth noting that, although for the composition range of $\mathrm{Mn}$ is higher than 2 mass\%, the cyclic transformation behavior can be initiated by providing extended time for nucleation of ferrite at higher temperatures (i.e. lower undercoolings); we also could have opted for selecting lower critical $T_{1}$ and $T_{2}$ temperatures for the cyclic treatment. However, when calculating the bainite start temperature for this range of composition using $B_{\mathrm{s}}(\mathrm{K})=$ $907.95-193.1 \mathrm{C}+02.4 \mathrm{C}^{2}-31.2 \mathrm{Mn}+10.36 \ln \left(d_{\gamma}\right)^{35}$ (where the $\mathrm{C}$ and Mn compositions are in mass $\%$ and $\mathrm{d}_{\gamma}$ is the austenite grain size in $\mu \mathrm{m}$ ), a high probability of bainite formation during cyclic treatment at the lower transformation temperatures is predicted. Since the kinetics and the mechanism of bainite formation in these alloys are controversial and different from those of the proeutectoid ferrite, ${ }^{36,37}$ the occurrence of bainite formation in the CPPT treatment would complicate the interpretation of the dilatometry curves in relation to the degree of austenite reversion.

\section{CONCLUSION}

In summary, the systematic simulations of the cyclic partial phase transformations using the LE model revealed a critical $\mathrm{Mn}$ concentration range of 1.5-2.5 mass\% in which the CPPT treatment can locally increase the effective Mn concentration in regions near the moving austenite-ferrite interface. This process suppresses ferrite formation via controlled mode switching in the interfacial Mn partitioning and creates a post-cyclic stagnant stage. This stage is absent in the CC and IC thermal routes as there is no reversion stage. The systematic experiments on the alloys subjected to the CPPT route are in good agreement with the LE model predictions and confirm the predicted critical $\mathrm{Mn}$ concentration range from 1.5 mass $\%$ to 2.5 mass $\%$ required to create a post-cyclic stagnant stage via designated segregation of Mn near the austeniteferrite interfaces.

\section{ACKNOWLEDGEMENTS}

The authors gratefully acknowledge the support of the ArcelorMittal research center in Metz, France. Prof. Wei XU acknowledges the financial support of the National Natural Science Foundation of China (Nos. 51574080 and 51722101).

\section{OPEN ACCESS}

This article is distributed under the terms of the Creative Commons Attribution 4.0 International License (http://creativecommons.org/licenses/by/4.0/), which permits unrestricted use, distribution, and reproduction in any medium, provided you give appropriate credit to the original author(s) and the source, provide a link to the Creative Commons license, and indicate if changes were made.

\section{REFERENCES}

1. C. Capdevila, J. Cornide, K. Tanaka, K. Nakanishi, and E. Urones-Garrote, Metall. Mater. Trans. A 42, 3719 (2011).

2. H. Luo, J. Shi, C. Wang, W. Cao, X. Sun, and H. Dong, Acta Mater. 59, 4002 (2011).

3. C. Wang, W. Cao, J. Shi, C. Huang, and H. Dong, Mater. Sci. Eng., A 562, 89 (2013).

4. R. Zhang, W.Q. Cao, Z.J. Peng, J. Shi, H. Dong, and C.X. Huang, Mater. Sci. Eng., A 583, 84 (2013).

5. W.W. Sun, Y.X. Wu, S.C. Yang, and C.R. Hutchinson, Scr. Mater. 146, 60 (2018).

6. J. Shi, X. Sun, M. Wang, W. Hui, H. Dong, and W. Cao, Scr. Mater. 63, 815 (2010). 
7. R. Ding, D. Tang, and A. Zhao, Scr. Mater. 88, 21 (2014).

8. J. Hu, L.-X. Du, G.-S. Sun, H. Xie, and R.D.K. Misra, Scr. Mater. 104, 87 (2015).

9. O. Bouaziz, S. Allain, C.P. Scott, P. Cugy, and D. Barbier, Curr. Opin. Solid State Mater. Sci. 15, 141 (2011).

10. B.C. De Cooman, Curr. Opin. Solid State Mater. Sci. 8, 285 (2004).

11. Y.K. Lee and C. Choi, Metall. Mater. Trans. A 31, 355 (2000).

12. S. Zaefferer, J. Ohlert, and W. Bleck, Acta Mater. 52, 2765 (2004).

13. E. De Moor, D.K. Matlock, J.G. Speer, and M.J. Merwin, Scr. Mater. 64, 185 (2011).

14. S. Lee, S.-J. Lee, and B.C. De Cooman, Scr. Mater. 65, 225 (2011).

15. D.T. Pierce, D.R. Coughlin, D.L. Williamson, J. Kähkönen, A.J. Clarke, K.D. Clarke, J.G. Speer, and E. De Moor, Scr. Mater. 121, 5 (2016).

16. F. HajyAkbary, J. Sietsma, G. Miyamoto, T. Furuhara, and M.J. Santofimia, Acta Mater. 104, 72 (2016).

17. K.S. Choi, Z. Zhu, X. Sun, E. De Moor, M.D. Taylor, J.G. Speer, and D.K. Matlock, Scr. Mater. 104, 79 (2015).

18. J. Zhu, R. Ding, J. He, Z. Yang, C. Zhang, and H. Chen, Scr. Mater. 136, 6 (2017).

19. Z.-D. Li, G. Miyamoto, Z.-G. Yang, and T. Furuhara, Metall. Mater. Trans. A 42, 1586 (2011).

20. Z. Dai, R. Ding, Z. Yang, C. Zhang, and H. Chen, Acta Mater. 144, 666 (2018)

21. M. Gouné, F. Danoix, S. Allain, and O. Bouaziz, Scr. Mater. 68, 1004 (2013).
22. H. Chen and S. van der Zwaag, Metall. Mater. Trans. A 48, 2720 (2017). https://doi.org/10.1007/s11661-016-3826-7.

23. H. Chen, B. Appolaire, and S. van der Zwaag, Acta Mater. 59, 6751 (2011).

24. H. Chen and S. van der Zwaag, Acta Mater. 61, 1338 (2013).

25. M.-I. Tzini, P. Sarafoglou, A. Stieben, G. Haidemenopoulos, and W. Bleck, Steel Res. Int. 87, 1686 (2016).

26. A. Borgenstam, L. Höglund, J. Agren, and A. Engström, J. Phase Equilib. 21, 269 (2000).

27. H. Chen and S. van der Zwaag, in TMP 2012-4th Int. Conf. Thermomechanical Process. Steels (Sheffield, 2012).

28. H. Chen and S. van der Zwaag, Comput. Mater. Sci. 49, 801 (2010).

29. J.O. Andersson, T. Helander, L. Höglund, P. Shi, and B. Sundman, Calphad 26, 273 (2002)

30. H. Chen, W. Xu, M. Goune, and S. van der Zwaag, Philos. Mag. Lett. 92, 547 (2012).

31. H. Farahani, W. Xu, and S. van der Zwaag, Metall. Mater. Trans. A 46, 4978 (2015).

32. M. Hillert, Scr. Mater. 46, 447 (2002).

33. H. Chen, R. Kuziak, and S. van der Zwaag, Metall. Mater. Trans. A 44, 5617 (2013).

34. H. Chen, E. Gamsjäger, S. Schider, H. Khanbareh, and S. van der Zwaag, Acta Mater. 61, 2414 (2013).

35. S. Kang, S. Yoon, and S.-J. Lee, ISIJ Int. 54, 997 (2014).

36. L.C.D. Fielding, Mater. Sci. Technol. 29, 383 (2013).

37. H.I. Aaronson and M.G. Hall, Metall. Mater. Trans. A 25, 1797 (1994). 